\title{
The Correlation between the Indonesian Version of Montreal Cognitive Assessment and Homocysteine Levels in Bataknese Male with Schizophrenia in Prof. DR. M. Ildrem Psychiatric Hospital Medan
}

\author{
Yoseva Hotnauli, Bahagia Loebis (D), M. Surya Husada*(D), Nazli Mahdinasari Nasution (i), Elmeida Effendy (D) \\ Department of Psychiatry, Faculty of Medicine, Universitas Sumatera Utara, Medan, Indonesia
}

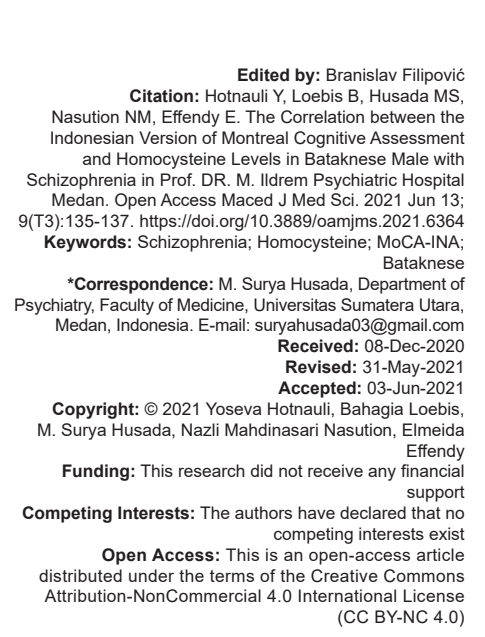

Abstract

BACKGROUND: The pathogenesis of schizophrenia and its mechanism is not convinced. Several studies indicate that schizophrenia pathogenesis can be related to changes at the cellular level. The studies show homocysteine in people with schizophrenia was significantly increased, abnormal homocysteine metabolism can lead to DNA methylation.

AIM: This study aimed to establish the correlation between the Indonesian Montreal Cognitive Assessment Version (MoCA-Ina) scores and homocysteine levels in males with Bataknese schizophrenia.

METHODS: This study is a numerical correlative analytic study with an approach to cross-sectional study; by evaluating the correlation between the MoCA-Ina scores and the level of homocysteine in males with Bataknese schizophrenia.

RESULTS: The median of the MoCA-Ina score in the study subjects was 22, with a minimum score of 18 and a maximum score of 26 . There was a significant correlation between the MoCA-Ina scores and Homocysteine levels $(p=0.001)$.

CONCLUSION: The interpretation obtained from this study is that the higher Homocysteine level, the lower the MoCA-Ina scores in Bataknese men with schizophrenia is.

\section{Introduction}

Schizophreniais a complexpsychiatric condition identified by impairment, apathy social alienation, and cognitive impairment in reality testing. Many factors contribute to the development of schizophrenia [1]. Factors that contribute to schizophrenia include genetic, biological, biochemical, psychosocial, socioeconomic status, stress, and drug abuse [2].

The pathogenesis of schizophrenia and its mechanism is not convinced. Several studies show that altered DNA methylation, mitochondrial dysfunction, impaired glutamate neurotransmitters, and decreased folic acid can be associated with the pathogenesis of schizophrenia. Those studies found that homocysteine levels in people with schizophrenia were significantly increased. Abnormal homocysteine metabolism can lead to abnormal DNA methylation. Homocysteine changes are a risk factor for schizophrenia pathogenesis and high homocysteine levels can be correlated with the morbidity and cognitive impairment of schizophrenia [1].
In a study by Moustafa et al. in 2014 in Australia, The increase in homocysteine in schizophrenia patients and affective disorders led to their cognitive impairment. There are several mechanisms by which homocysteine may be biologically linked to psychiatric disorders. It has been found that homocysteine interacts with the receptor of N-MethylD-Aspartate (NMDA), initiating oxidative stress-inducing apoptosis, inducing mitochondrial dysfunction, and inducing vascular damage [3]. It has been found that homocysteine is an independent risk factor for cognitive dysfunction. Studies of individuals with a wide range of cognitive impairments have consistently shown increased plasma homocysteine and decreased enzymatic cofactors involved in methionine and homocysteine metabolism. Plasma total homocysteine also appears to be the most consistent tissue marker in the cofactor of nutrient deficiency, as well as cognitive performance in the elderly [4], [5], [6].

Deng et al.'s 2018 study in China, a significant increase in serum homocysteine can be used as a laboratory diagnostic tool to assess the severity of cognitive dysfunction in first-episode of schizophrenia patients. Severe cognitive impairment in schizophrenia patientsisstatedinthisstudy. Previousstudieshaveshown that 5-hydroxytryptamine and frontal lobe dopamine 
biosynthesis may be affected by serum homocysteine, which later affects frontal lobe activity. Prefrontal hypofunction is associated with cognitive impairment. This is why the increase in serum homocysteine in the patients with schizophrenia correlates with cognitive function. Subjects with first-episode schizophrenia with increased serum homocysteine of more than $15 \mu \mathrm{mol} / \mathrm{L}$ in this study, significantly had serum homocysteine levels and decreased cognitive function than subjects who had homocysteine levels $<15 \mu \mathrm{mol} / \mathrm{L}(p<0.05)$ [1].

Based on the differences in previous studies, Miller et al. [4] stated that there was no significant relationship between homocysteine levels and cognitive impairment, while studies by Moustafa et al. and Deng et al. stated that increased homocysteine contributed to disorders of cognitive, but the same research has never been conducted in Indonesia. Thus, through this study, the authors would like to know the correlation between the Indonesian Version of the Montreal Cognitive Assessment (MoCA-Ina) Score and homocysteine levels in Bataknese men with schizophrenia.

\section{Methods}

This research is a numerical correlative analytic study with a cross-sectional study approach which assesses the correlation between MoCA-Ina scores and Homocysteine levels of Bataknese men with schizophrenia. The sample in this study was Bataknese men with schizophrenia at the Polyclinic of RSJ, Prof. Dr. M. Ildrem Medan who met the inclusion criteria and the sampling was by means of nonprobability sampling, consecutive sampling type. The inclusion criteria in this study were Bataknese men with schizophrenia who had been diagnosed based on PPDGJ III, age between 20 and 40 years, duration of illness $1-5$ years, total PANSS score 60-80, understood Indonesian, were willing to be were interviewed, received antipsychotic medication risperidone $4 \mathrm{mg} /$ day/oral in divided doses, the latest education was at least Junior High School (SMP) or equivalent, smoking frequency $\leq 10$ cigarettes/day (light smoker), whereas the in this study, the exclusion criteria were general medical conditions and/or other comorbidities, history of use of alcohol and other substances, history of use of supplements containing Vitamin B (B6, B9, B12). This research was conducted from July to September 2019, and the total sample was 49 participants.

Data analysis, before data analysis were carried out data normality test was carried out using the Shapiro-Wilk test, then checking the linearity assumption by making a scatter chart. The data were evaluated in order to obtain the correlation value $(r)$. The data analysis was conducted using the Pearson Correlation test for data that were normally distributed. Data that were not normally distributed were analyzed using the Spearman correlation test. The collection and interpretation of data were carried out with the assistance of the software Statistical Package for Social Sciences.

\section{Results}

In this study, demographic characteristics Table 1 were obtained based on age group, with a mean of 30.61 years with a standard deviation of 5.89 , participants in the age of 20-30 years group were 24 people (48.9\%), and participants in the age of 31-40 years group as many as 25 people $(51.5 \%)$. Based on the level of education, junior high school grade were 18 people $(36.7 \%)$, senior high school grade were 28 people $(57.2 \%)$, college graduates were three people (6.1\%). On marital status, participants with married status were 11 people $(22.5 \%)$. Likewise with employment status, subjects who did not work were 29 people (59.2\%). Based on the long sick, the median was found to be 3.00 with the minimummaximum (1-5). The mean PANSS score was 74.82 and the standard deviation was 3.26.

Table 1: Distribution of research participants based on demographic characteristics

\begin{tabular}{llll}
\hline Demographic characteristics & $\begin{array}{l}\text { Mean } \pm \text { SB } \\
\text { Median (min-max) }\end{array}$ & $\mathrm{n}$ & $(\%)$ \\
\hline Age & & 24 & $(48.9)$ \\
$20-30$ & & 25 & $(51.1)$ \\
$31-40$ & & 18 & $(36.7)$ \\
Level of education & & 28 & $(57.2)$ \\
$\quad$ Junior high school & 3 & $(6.1)$ \\
$\quad$ Senior high school & & \\
$\quad$ University & & 11 & $(22.5)$ \\
Marriage status & 38 & $(77.5)$ \\
$\quad$ Married & & & \\
$\quad$ Not married & & 20 & $(40.8)$ \\
Job status & & & \\
$\quad$ Work & & & \\
$\quad$ Does not work & & & \\
Long sick & & & \\
PANSS score & $3.00(1-5)$ & & \\
\hline
\end{tabular}

The mean homocysteine levels in the participants of this study were obtained at 25.46 and the standard deviation is 9.17. The median of the MoCA-Ina scores in the study participants was 22 , with a minimum score of 18 and a maximum score of 26 . In the Table 2 the correlation between the MoCA-Ina scores and homocysteine levels, after normalizing the data distribution, was tested for correlation. Pearson, where previously checked the linearity assumption by making a scatter chart. From the analysis results obtained $p=0.001$ which indicates that there is a significant correlation between the MoCA-Ina scores and homocysteine levels. The Pearson correlation value of -0.754 indicates a negative correlation with a strong correlation strength, this indicates that the higher the homocysteine level, the lower the MoCA-Ina score.

\section{Discussion}

In this study, only male participants were included in the study. This is because, in a 2002 study by 
Table 2: Results of the Pearson correlation test analysis between MoCA-Ina score and Homocysteine levels in Bataknese men with schizophrenia

\begin{tabular}{ll}
\hline & Homocysteine levels \\
\hline MoCA-Ina score & $r=-0.754$ \\
& $p=0.001$ \\
& $n=49$ \\
\hline MoCA-Ina: Indonesian version of the montreal cognitive assessment. &
\end{tabular}

Levine et al. In Israel, it was found that in younger male patients with schizophrenia, homocysteine levels were higher. It is well known that the onset of schizophrenia is earlier in male patients than in women and that the disease worsens chronically more often in younger men. In a study by Yang et al. in 2015 in China, it was found that the prevalence of age-related hyperhomocysteinemia in men was substantially greater than women [7], [8].

In a study by Di Lorenzo et al. in 2015 in Italy, it was found that in the group of people with schizophrenia, there was a significant rise in homocysteine levels with duration of illness $>1$ year compared to the group of people with schizophrenia with duration of illness $<1$ year ( $X^{2}$ test, $p=0.02$ ). The study of Narayan et al. in India in 2014, found that there was a statistically significant relationship between homocysteine and duration of illness (Spearman's analysis test $p=0.2866$ $p=0.0004$; Kendall score $=940, S E=330, p=0.0045$ ). Meanwhile, in the 2010 Mabrouk study in Tunisia, it was found that in patients with schizophrenia, homocysteine plasma levels were not associated with duration of illness $(r=-0.04, p=0.78)$ [9], [10], [11].

In the study conducted by Ma et al. 2009 in Hong Kong, it was found that there was no significant relationship between the PANSS score and serum homocysteine level $(r=0.066, p=0.303)$. The same thing was found in the study of Narayan et al. in 2014 that the PANSS positive symptom score of schizophrenia was not significantly correlated with homocysteine levels $(r=0.007, p=0.9)$, but PANSS negative scores had a significant correlation with homocysteine levels $(r=0.7 p=<0.001)$ [11], [12].

In this study, the correlation between the MoCAIna score and homocysteine levels in Bataknese men with schizophrenia obtained $p=0.001$ which indicates that the correlation between the MoCA-Ina score and homocysteine levels is significant. The Pearson correlation value of $-0,754$ indicates a negative correlation with strong correlation strength. This shows that the higher the homocysteine level, the lower the MoCA-Ina score is. The results of this study are in accordance with a study conducted by Moustafa et al. in 2014 in Australia which stated that increased homocysteine could also contribute to cognitive impairment. It was found that homocysteine interacted with the NMDA receptor, initiating oxidative stress-inducing apoptosis, trigger mitochondrial dysfunction, and caused vascular damage. In a study conducted by Moustafa et al. in 2018 in China, it was found that the first episode schizophrenic subjects had significantly increased serum homocysteine more than $15 \mu \mathrm{mol} / \mathrm{L}$ and lower cognitive function than participants who had homocysteine levels less than $15 \mu \mathrm{mol} / \mathrm{L}(p<0.05)$ [1], [3].

\section{Conclusion}

The interpretation obtained from this study is that the higher Homocysteine level, the lower the MoCAIna scores in Bataknese men with schizophrenia.

\section{References}

1. Jing D, Mao X, Zhi L, Xinyuan L. Cognitive ability and the level of serum homocysteine correlations in first-episode schizophrenia subjects. J Psychiatry Brain Sci. 2018;3(2):1-6. https://doi. org/10.20900/jpbs.20180003

2. Zahnia S, Sumekar DW. Epidemiological study of schizophrenia. Majority. 2016;5(4):160-6.

3. Moustafa AA, Hewed DH, Eissa AM, Frydecka D, Misiak B Homocysteine levels in schizophrenia and affective disordersfocus on cognition. Front Behav Neurosci. 2014;8(343):1-10. https://doi.org/10.3389/fnbeh.2015.00081

\section{PMid:25339876}

4. Miller AL. The methionine-homocysteine cycle and its effects on cognitive diseases. Altern Med Rev. 2003;8(1):7-19.

PMid:12611557

5. Teunissen CE, van Boxtel MP, Jolles J, Vente JD, Vreeling F, Verhey $\mathrm{F}$, et al. Homocysteine in relation to cognitive performance in pathological and non-pathological conditions. Clin Chem Lab Med. 2005;43(10):1089-95. https://doi.org/10.1515/cclm.2005.190 PMid:16197303

6. Sadock BJ, Sadock V, Ruiz P. In: Sadock BJ, Sadock VA, Ruiz P, editors. Kaplan and Sadock's Comprehensive Textbook of Psychiatry. 10 ${ }^{\text {th }}$ ed., Vol. 1, 2. New York: Wolters Kluwer; 2017. p. 2751. https://doi.org/10.4067/s0717-92272002000300011

7. Levine J, StahIZ, SelaBA, GavendoS, Ruderman V,BelmakerRH Elevated homocysteine levels in young male patients with schizophrenia. Am J Psychiatry. 2002;159(10):1790-2. https:// doi.org/10.1176/appi.ajp.159.10.1790

PMid:12359692

8. Yang B, Fan S, Zhi X, Wang $\mathrm{Y}$, Wang $\mathrm{Y}$, Zheng $\mathrm{Q}$, et al Prevalence of hyperhomocysteinemia in China: A systematic review and meta-analysis. Nutrients. 2015;7(1):74-90. https:// doi.org/10.3390/nu7010074

PMid:25551247

9. Mabrouka H, Doukia W, Mechrib A, Younesa MK, Omezzinec A Bouslamac A, et al. Hyperhomocysteinemia and schizophrenia: Case control study. Lencephale. 2011;37(4):308-13. PMid:21981892

10. Di Lorenzo R, Amoretti A, Baldini S, Soli M, Landi G, Pollutri G, et al. Homocysteine level in schizophrenia patients newly admitted to an acute psychiatric ward. Acta Neuropsychiatrica. 2015;27(6):336-44. https://doi.org/10.1017/neu.2015.30 PMid:26017629

11. Narayan SK, VermanA, KattimaniS,Ananthanarayanan $\mathrm{PH}, \mathrm{Adithan}$ C. Plasma homocysteine levels in depression and schizophrenia in South Indian Tamilian population. Indian J Psychiatry. 2014;56(1):46-53. https://doi.org/10.4103/0019-5545.124746 PMid:24574558

12. Ma YY, Shek CC, Wong MC, Yip KC, Ng RM, Nguyen DG, et al. Homocysteine level in schizophrenia patients. Aust N Z J Psychiatry. 2009;43(8):760-5.

PMid:19629798 